\title{
Investigation into the Reactivity between Various Amino Acids and Oxygen-Derived Free Radicals by Use of the ESR Spin Trapping Method
}

\author{
Toshikazu Yoshikawa, ${ }^{*}$ Shuji TaKahashi, Toru Tanigawa, \\ Yuji NaIto, Hiroshi ICHIKawa, Hirohisa TaKano, \\ and Motoharu Kondo
}

First Department of Medicine, Kyoto Prefectural

University of Medicine, Kamigyo-ku, Kyoto 602, Japan

(Received April 5, 1991)

\begin{abstract}
Summary Considerable attention has recently centered on the possible role of oxygen-derived free radicals in protein damage and degradation. In the present study, we investigated the reactivity of amino acids with superoxide and hydroxyl radical by an electron spin resonance assay using 5,5-dimethyl-1-pyrroline-N-oxide (DMPO) as a spin trap. The intensity of the DMPO-OOH spin adduct generated from the hypoxanthine-xanthine oxidase system decreased in the presence of some amino acids such as cysteine and methionine at a final concentration of 1-10 $\mathrm{mM}$. However it was not influenced by the presence of other amino acids at the same final concentrations. The relative intensity of the DMPO-OH spin adduct generated from the Fenton reaction significantly decreased in the presence of various amino acids, especially aromatic amino acids, sulfur-containing amino acids, and histidine. The inhibition of DMPO$\mathrm{OH}$ signal was not due to the inhibition of the Fenton reaction, but due to the competition of the amino acid with DMPO. In the early step of protein damage by active oxygen species, such amino acids residues are attacked.
\end{abstract}

Key Words: amino acids, superoxide, hydroxyl radical, ESR spin trapping method, protein damage

Oxygen-derived free radicals have recently been implicated in a number of biochemical and chemical reactions in the body. Their toxicity has a major impact on most biomolecules, such as lipids, nucleic acids, proteins, carbohydrates, and

*To whom correspondence should be addressed. 
other low molecular weight compounds. Lipid peroxidation induced by free radical reactions has been well studied and is believed to be one of the major causes of cell damage and injuries to cell membranes. In contrast, the reactions of proteins with various free radicals have not been so extensively studied. However, it has recently been clarified that amino acids, peptides, and proteins are vulnerable to attack by free radicals $[1,2]$. And a considerable number of reports $[3,4]$ now suggest that oxidative modification of protein and proteolysis may be closely linked. The first step in the proteolytic degradation of enzymes or proteins is an oxygen-dependent modification of some amino acid residues in the protein [5-8]. So, in the present study, in order to investigate the protein damage by free radicals, we first measured the reactivity of amino acids with superoxide anion radical $\left(\mathrm{O}_{2}^{\bar{\alpha}}\right)$ and hydroxyl radical $\left({ }^{\circ} \mathrm{OH}\right)$ by means of an electron spin resonance (ESR) assay using 5,5-dimethyl-1-pyrroline-N-oxide (DMPO) as a spin trap.

\section{MATERIALS AND METHODS}

The following 23 amino acids were tested (all products were supplied by Morishita Pharmaceutical Co., Ltd., Tokyo): alanine, arginine, asparagine, aspartic acid, cysteine, glycine, glutamic acid, glutamine, histidine, hydroxylproline, isoleucine, leucine, lysine, methionine, ornithine, phenylalanine, proline, serine, taurine, threonine, tryptophan, tyrosine, and valine. DMPO, hypoxanthine (HX), xanthine oxidase (XO), and diethylenetriaminepentaacetic acid (DETAPAC) were obtained from Sigma Chemical Co., St. Louis, MO, and hydrogen peroxide and dimethyl sulfoxide (DMSO), from Wako Pure Chemical Ind., Osaka.

Superoxide-scavenging activity was measured by the inhibition of the ESR signal of the DMPO-OOH spin adduct generated from the HX-XO system in the presence of DMPO [9]. The reaction mixture was previously mixed with $50 \mathrm{~mm}$ sodium phosphate buffer solution ( $\mathrm{pH} 7.4$ ), $0.5 \mathrm{~mm} \mathrm{HX}, 0.1 \mathrm{~mm}$ DETAPAC, $0.1 \mathrm{M}$ DMPO, and various concentrations of standard SOD or sample in a small tube. The reaction was started by the addition of $5 \mathrm{mU} / \mathrm{ml} \mathrm{XO}$. (One unit of enzyme activity is defined as the amount of enzyme required to convert $1 \mathrm{~mol}$ of xanthine to uric acid per minute at $37^{\circ} \mathrm{C}$.) An aliquot of reaction mixture was then transferred into a quartz cell, and recording of the ESR spectrum was started 2 min after the addition of XO. The intensity of the DMPO-OOH signal was measured as the ratio to the intensity of the $\mathrm{Mn}^{2+}$ signal. The reactions were carried out at $25^{\circ} \mathrm{C}$.

Hydroxyl radical-scavenging activity was measured by the inhibition of the ESR signal of the DMPO-OH spin adduct generated from the Fenton reaction in the presence of DMPO [10]. The reaction mixture contained $50 \mathrm{mM}$ sodium phosphate buffer ( $\mathrm{pH} 7.4$ ), $50 \mu \mathrm{M}$ ferrous sulfate, $0.1 \mathrm{mM}$ DETAPAC, $1 \mathrm{mM}$ or 10 $\mathrm{mM}$ DMPO, and $1 \mathrm{mM}$ hydrogen peroxide in a total volume of $200 \mu \mathrm{l}$. The ferrous sulfate solution and then DMPO and a sample solution were added to the phosphate buffer containing DETAPAC. The reaction was started by the addition of hydrogen peroxide within 1 min after the addition of ferrous sulfate. We call 
this type of experiment 'preaddition' to differentiate it from the 'postaddition' experiments where the test substance was added only after the addition of hydrogen peroxide. The reactions were carried out at $25^{\circ} \mathrm{C}$. The ESR spectral recording was started $1 \mathrm{~min}$ after the addition of hydrogen peroxide.

To distinguish whether the inhibition of the DMPO-OH signal is due to the competition of the test substance with DMPO or to inhibition of the Fenton reaction, at least two DMPO concentrations were used [10]. That is to say, if the inhibition by a substance is totally due to the inhibition of Fenton reaction, the inhibition rate must be dependent on the absolute concentration of the substance, and independent of the DMPO concentration. On the contrary, if the inhibition is totally due to competition between DMPO and the test substance, the inhibition rate should be dependent on the relative concentration of the substance vs. DMPO.

The hydroxyl radical-scavenging activity of the samples was expressed as the relative value of the second-order rate constant of the reaction with the hydroxyl radical against that of DMPO (Eq. (1)).

$$
R=\frac{k_{\mathrm{x}}}{k_{\mathrm{DMPO}}} \text {. }
$$

Let $V$ and $v$ represent the rate of spin trapping in the absence and presence of the substance, and $[\mathrm{x}]$ and [DMPO] are the molar concentration of the substance and DMPO, respectively. The intensity of the ESR signal represents the formation of the adduct and the rate of spin trapping. Thus, Eq. (2) can be induced.

$$
\frac{I}{i}=\frac{V}{v}=\frac{k_{\mathrm{x}}[\mathrm{x}]}{k_{\mathrm{DMPO}}[\mathrm{DMPO}]}+1,
$$

where $I$ and $i$ are the ESR signal intensity of DMPO-OH in the absence and presence of the substance, respectively. By use of Eq. (2), Eq. (1) can be transformed to Eq. (3).

$$
R=\left(\frac{I}{i}-1\right) \cdot \frac{[\mathrm{DMPO}]}{[\mathrm{x}]}=\frac{F \cdot[\mathrm{DMPO}]}{(1-F) \cdot[\mathrm{x}]}
$$

where $F$ is the inhibition rate of the DMPO-OH signal [10].

To express the reactivity of a solution, we used $R^{\prime}$ instead of $R$ [10]. $R^{\prime}$ corresponds to $R$ in Eq. (3), from which the concentration factor [x] is omitted (Eqs. (4), (5)).

$$
\begin{gathered}
R^{\prime}=\frac{F}{1-F} \cdot[\mathrm{DMPO}], \\
R^{\prime}=R[\mathrm{x}] .
\end{gathered}
$$

One unit was defined as the scavenging activity comparable to that of one mole of $\mathrm{DMPO}$, and the solution which has $1 \mathrm{U} /$ liter activity will scavenge ${ }^{\circ} \mathrm{OH}$ as much as a one molar DMPO solution. $R$ is the specific value of the substance, and $R^{\prime}$ is 
the specific value for a solution [10].

\section{RESULTS AND DISCUSSION}

\section{Reactivity with superoxide}

Although the DMPO-OOH signal was slightly suppressed with cysteine, glutamic acid, arginine, methionine, and histidine, it required a relatively high concentration of 1-10 mM. Even with cysteine (Fig. 1), with which the greatest suppression was observed, the 50\% suppression concentration was $2.3 \mathrm{~mm}$. One mole of cysteine was equivalent to $485 \mathrm{U}$ of SOD. Glutamic acid inhibited XO activity at a final concentration of $5 \mathrm{~mm}$, which was shown by decreased uric acid formation. Taking this into consideration, suppression of the DMPO-OOH signal by glutamic acid did not mean superoxide-scavenging activity of glutamic acid. Arginine, methionine, and histidine, which did not inhibit XO activity, had slight superoxide-scavenging activity. But the precise mechanism of their action is still unknown. Even with the high concentrations of $10-100 \mathrm{~mm}$, the other amino acids had practically almost no effect on the DMPO-OOH signal, and it was clear that the reactivity between superoxide with amino acids was not very high.

\section{Reactivity with hydroxyl radical}

All 23 amino acids investigated by preaddition suppressed the DMPO-OH

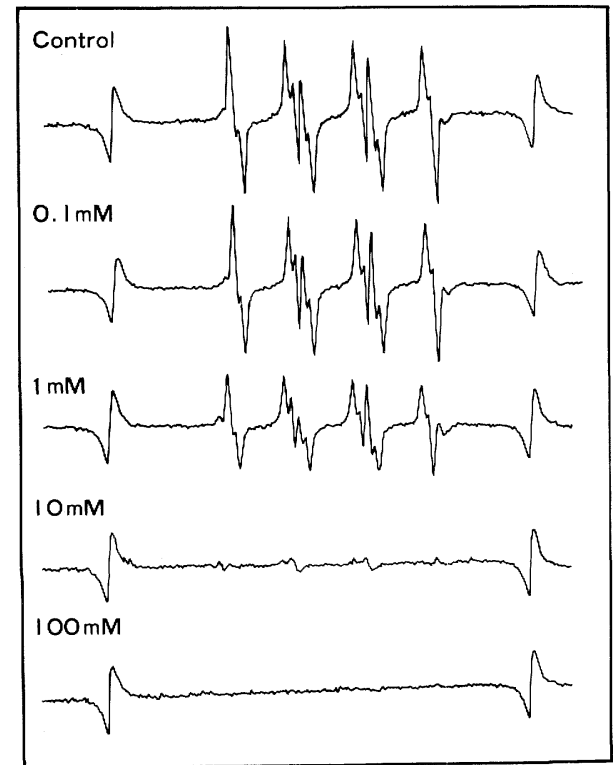

Fig. 1. ESR spectra obtained when hypoxanthine was incubated with xanthine oxidase in the presence of $0.1 \mathrm{mM}$ DMPO with or without cysteine. 
signal concentration dependently, and the effect clearly varied according to the various amino acids (Table 1). Strong suppression was observed with aromatic amino acids, sulfur-containing amino acids, and histidine among others (Fig. 2). If the inhibition by an amino acid is totally due to the inhibition of the Fenton reaction, the inhibition rate must be dependent on the absolute concentration of the amino acid [10]. But the inhibition rate by each amino acid was dependent on the relative concentration of every amino acid vs. DMPO, so this inhibition was not due to the Fenton reaction itself, but due to competition between the amino acids and DMPO. As an example, the effect of the sulfur-containing amino acids, methionine and cysteine, on the DMPO-OH signal is shown in Fig. 3. $R$ and $R^{\prime}$ values determined by the Tanigawa method [10] are shown in Table 2.

On the other hand, none of the amino acids had practically any effect on the DMPO-OH signal with postaddition (Table 1). This indicates that no amino acid induced the decay of an already generated DMPO-OH spin adduct. Therefore, the suppression of the DMPO-OH signal observed with preaddition was ultimately caused by the competition between the various amino acids and DMPO.

In experiments in which proteins were damaged by radiation exposure and

Table 1. Effect of amino acids on DMPO-OH signal generated from the Fenton reaction at $1 \mathrm{mM}$ DMPO.

\begin{tabular}{lccc}
\hline Amino acids & $\begin{array}{c}\text { Final conc. } \\
(\mathrm{mM})\end{array}$ & $\begin{array}{c}\text { Preaddition } \\
(\%)\end{array}$ & $\begin{array}{c}\text { Postaddition } \\
(\%)\end{array}$ \\
\hline Alanine & 1 & 99.9 & 103.4 \\
Arginine & 1 & 65.0 & 99.1 \\
Asparagine & 1 & 90.0 & 93.7 \\
Aspartic acid & 0.32 & 74.5 & 91.6 \\
Cysteine & 1 & 5.9 & 101.0 \\
Glycine & 1 & 100.8 & 96.6 \\
Glutamic acid & 1 & 75.4 & 101.8 \\
Glutamine & 1 & 85.2 & 99.8 \\
Histidine & 1 & 6.6 & 96.6 \\
Hydroxyproline & 1 & 83.7 & 93.9 \\
Isoleucine & 1 & 19.9 & 92.6 \\
Leucine & 1 & 17.9 & 100.3 \\
Lysine & 1 & 57.9 & 93.8 \\
Methionine & 6.5 & 102.1 \\
Ornithine & 1 & 78.6 & 92.7 \\
Phenylalanine & 1 & 0.0 & 94.2 \\
Proline & 1 & 78.2 & 96.1 \\
Serine & 1 & 74.3 & 97.5 \\
Taurine & 1 & 89.5 & 99.9 \\
Threonine & 1 & 83.2 & 102.2 \\
Tryptophan & 1 & 5.8 & 96.1 \\
Tyrosine & 1 & 54.8 & 90.5 \\
Valine & 0.2 & 54.7 & 95.6 \\
\hline Comparis & 1 & &
\end{tabular}

Comparison between preaddition and postaddition experiments of amino acids. \% ratio to the value in the absence of the amino acid. 


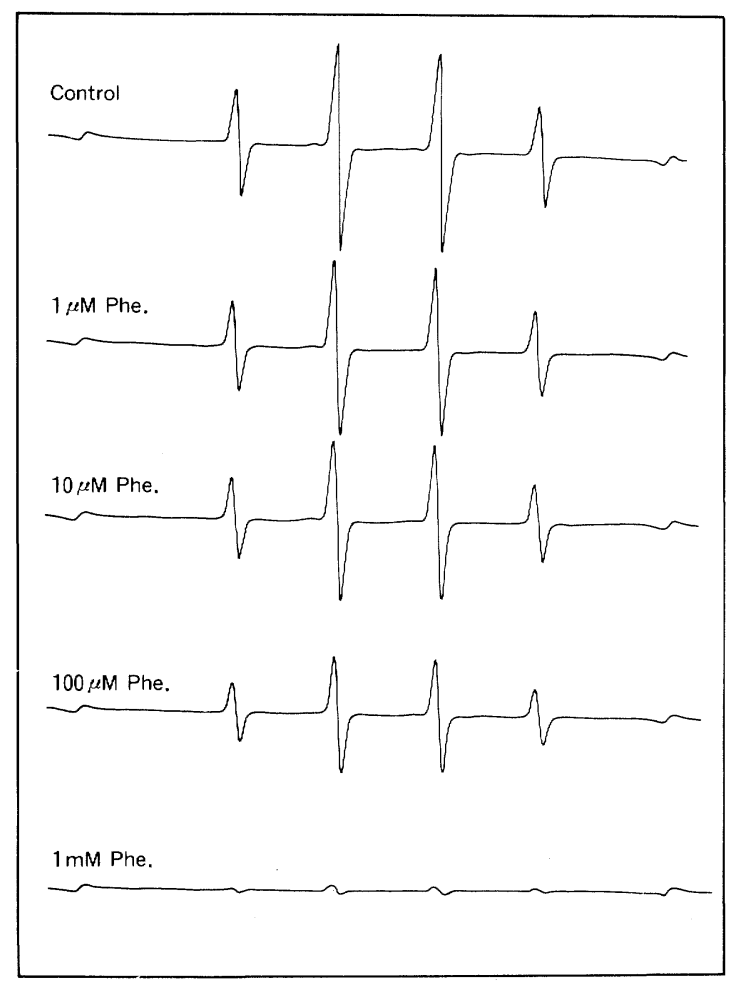

Fig. 2. Effect of phenylalanine on the DMPO-OH signal obtained from the Fenton reaction at $1 \mathrm{mM}$ DMPO.

free amino acids were obtained from them by hydrolysis and analyzed, histidine, tyrosine, phenylalanine, tryptophan, cysteine, methionine, and lysine showed a marked decrease, whereas amino acids with an alkyl group on a side chain, including alanine, were said to be relatively stable [11]. Damage caused by the radiation exposure was believed to originate indirectly from hydroxyl radicals produced from water molecules after extraction of hydrogen. That report is in relatively good agreement with the reactivity between single amino acids and hydroxyl radicals in our present experiment. As for the reactivity with hydroxyl radical, it's highly possible that amino acid residues in proteins and free amino acids may show a similar tendency. Although studies may be needed with regard to the effects of protein conformation, $\mathrm{pH}$ of reaction solution, and so on, we felt the present data could be used as a reference after taking into consideration the mechanism of protein damage caused by active oxygen species.

As far as cysteine's reactivity with ${ }^{\circ} \mathrm{OH}$ is concerned, when the reactivity of glutathione and $\mathrm{N}$-acetyl cysteine with ${ }^{\circ} \mathrm{OH}$ was compared, the $R$ values were virtually the same; and their reactivity was, for the most part, thought to be derived from the thiol group. The thiol group is closely involved in the action of many 


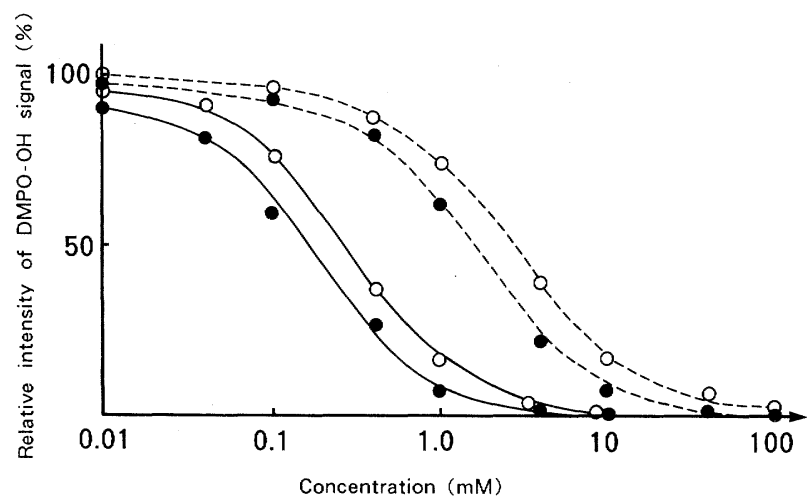

Fig. 3. Effect of the sulfur-containing amino acids on the DMPO-OH signal obtained from the Fenton reaction at $1 \mathrm{mM}$ or $10 \mathrm{~mm}$ DMPO. $\bigcirc \mathrm{O}$, Methionine at $10 \mathrm{~mm}$ DMPO; $\bigcirc \cdots \cdots$. , methionine at $1 \mathrm{mM}$ DMPO; $\bullet$, cysteine at $10 \mathrm{~mm}$ DMPO; $\cdots \cdots \cdot$, cysteine at $1 \mathrm{mM}$ DMPO. The ordinate expresses the ratio (as a percentage) of the value obtained in the presence of the amino acid to that in its absence.

Table 2. Reactivity of amino acids with hydroxyl radical.

\begin{tabular}{|c|c|c|c|}
\hline Amino acids & $R$ & $\begin{array}{l}\text { Serum level }{ }^{\mathrm{a}} \\
(\mu \mathrm{M})\end{array}$ & $\begin{array}{c}R^{\prime} \\
(\mathrm{mU} / \text { liter })\end{array}$ \\
\hline Alanine & 0.05 & 419 & 0.021 \\
\hline Arginine & 0.54 & 89 & 0.048 \\
\hline Asparagine & 0.10 & 49 & 0.005 \\
\hline Aspartic acid & 1.07 & 13 & 0.014 \\
\hline Cysteine & 6.81 & 118 & 0.804 \\
\hline Glycine & 0.01 & 230 & 0.002 \\
\hline Glutamic acid & 0.33 & 60 & 0.020 \\
\hline Glutamine & 0.17 & 645 & 0.110 \\
\hline Histidine & 4.88 & 89 & 0.434 \\
\hline Hydroxyproline & 0.32 & 20 & 0.006 \\
\hline Isoleucine & 3.33 & 84 & 0.280 \\
\hline Leucine & 2.04 & 160 & 0.326 \\
\hline Lysine & 0.73 & 198 & 0.145 \\
\hline Methionine & 3.72 & 32 & 0.119 \\
\hline Ornithine & 0.21 & 65 & 0.018 \\
\hline Phenylalanine & 14.13 & 65 & 0.918 \\
\hline Proline & 0.28 & 239 & 0.067 \\
\hline Serine & 0.35 & 114 & 0.040 \\
\hline Taurine & 0.04 & 162 & 0.006 \\
\hline Threonine & 0.55 & 146 & 0.080 \\
\hline Tryptophan & 5.75 & 60 & 0.345 \\
\hline Tyrosine & 4.12 & 72 & 0.297 \\
\hline Valine & 0.83 & 252 & 0.209 \\
\hline
\end{tabular}

Vol. 11, No. 3, 1991 
enzymes and hormones and in the functions of other biologically active proteins, and the fact that it is involved in many physiologic and biochemical reactions such as oxidative phosphorylation, photosynthesis, muscular contraction, cell division, and mitochondrial membrane permeability has been well founded. The fact that the thiol group has high reactivity with active oxygen species, especially with ${ }^{\circ} \mathrm{OH}$, shows that it may play an important role in modifications by active oxygen species, in inactivation of enzymes, and in various physiologic and biochemical processes.

In addition, there are several reports $[7,12]$ about inactivation of glutaminesynthesizing enzymes by oxidative modification of histidine residues, and other report [8] about protein damage caused by oxidation of methionine residues. In our present study, the $R$ values of histidine and methionine were 4.88 and 3.72 (Table 2), respectively; and both were highly reactive with ${ }^{\circ} \mathrm{OH}$, which is consistent with the findings mentioned above.

On the other hand, when various amino acids reacted with active oxygen species, secondary radical are generated [14]. In our measurement system also, secondary radicals were detected with high concentrations $(10-100 \mathrm{mM})$ of cysteine and methionine. Although secondary radicals derived from amino acid residues are generated in the early stages of protein damage by active oxygen species, it is said that their life span is extremely short, for they change rapidly and disappear. However, future studies will be needed with regard to the details of the process from oxidation of amino acid residues to protein degeneration.

\section{Active oxygen species-scavenging activity of amino acid preparations used in infusion solutions}

Finally, the reactivity with $\mathrm{O}_{2}^{-}$and ${ }^{\circ} \mathrm{OH}$ of an amino acid preparation that is actually used clinically (Moripron, Morishita Pharmaceuticals, Tokyo) was investigated. In the case of the DMPO-OOH signal, even with a 10-fold dilution of the preparation concentration only a $22.9 \%$ suppression was observed, and virtually no suppression with a 100-fold dilution. However, in the case of the DMPO-OH signal, it was almost completely suppressed with a 10 -fold dilution of the preparation concentration and a $10 \mathrm{mM}$ concentration of DMPO, and with a 100 -fold dilution and $1 \mathrm{mM}$ DMPO. The $R^{\prime}$ value calculated from the amino acid components of Moripron was over $1,700 \mathrm{mU} /$ liter, which is about 16 times that of human serum [10]. Therefore the $R^{\prime}$ value of human serum just after $500 \mathrm{ml}$ of this amino acid preparation is intravenously administered is about twice as large as that of human serum before the administration. Naturally the reactivity of this amino acid preparation with $\cdot \mathrm{OH}$ was considered, and although the components were not chosen for that reason, if amino acid preparations are made that focus on amino acids with high reactivity, it's possible that they can be applied to the prevention of tissue injury caused by free radicals.

The reactivity of various amino acid with $\mathrm{O}_{2}^{\overline{-}}$ and ${ }^{\circ} \mathrm{OH}$ was investigated by the ESR spin trap method. Because of the breakup of higher order structures, proteins oxidized in vivo are easily hydrolyzed by proteases, which speeds up the metabolic 
turnover of proteins. And although this has been discussed in relation to aging, we found in this study that $\mathrm{OH}$ was stronger oxidant to amino acids, and that aromatic amino acids, sulfur-containing amino acids, and histidine were fairly easy targets of oxidation. In the future, along with the task of clarifying the mechanism of protein damage caused by active oxygen species, we have made plans based on this data to continue investigating the possibility of preventing free radical-induced protein damage by the use of amino acids.

\section{REFERENCES}

1. Stadman, E.R. (1986): Oxidation of proteins by mixed-function oxidation systems. Trends Biol. Sci., 11, 11-12.

2. Davies, K.J.A. (1986): Intracellular proteolytic systems may function as secondary antioxidant defenses: A hypothesis. Free Radical Biol. Med., 2, 155-173.

3. Davies, K.J.A. (1987): Protein damage and degradation by oxygen radicals. J. Biol. Chem., 262, 9895-9901.

4. Rivett, A.J. (1985): Preferential degradation of the oxidatively modified form of glutamine synthetase by intracellular mammalian proteases. J. Biol. Chem., 260, 300-305.

5. Levine, R.L. (1983): Oxidative modification of glutamine synthetase. I. Inactivation is due to loss of one histidine residue. J. Biol. Chem., 258, 11823-11827.

6. Levine, R.L. (1983): Oxidative modification of glutamine synthetase. II. Characterization of the ascorbate model system. J. Biol. Chem., 258, 11828-11833.

7. Fucci, L., Oliver, C.N., and Coon, M.J. (1983): Inactivation of key metabolic enzymes by mixed-function oxidation reactions: Possible implication in protein turnover and aging. Proc. Natl. Acad. Sci. U.S.A., 80, 1521-1525.

8. Matheson, N.R., Wong, P.S., Shuyler, M. (1981): Interaction of human $\alpha$-1-proteinase inhibitor with neutrophil myeloperoxidase. Biochemistry, 20, 331-336.

9. Miyagawa, H., Yoshikawa, T., Tanigawa, T., Yoshida, N., Sugino, S., Kondo, M., Nishikawa, H., and Kohno, M. (1988): Measurement of serum superoxide dismutase activity by electron spin resonance. J. Clin. Biochem. Nutr., 5, 1-7.

10. Tanigawa, T. (1990): Determination of hydroxyl radical scavenging activity by electron spin resonance. J. Kyoto Pref. Univ. Med., 99, 133-143.

11. Rokushika, S. (1988): Radiation inactivation of enzymes. Tanpakushitsu Kakusan Kohso, 33, 3087-3093 (in Japanese).

12. Armstrong, M.D., and Stave, U. (1973): The serum levels of amino acids in humans. Metabolism, 22, 561-567.

13. Farber, J.M., and Levine, R.L. (1986): Sequence of a peptide susceptible to mixed-function oxidation. Probable cation binding site in glutamine synthetase. J. Biol. Chem., 261, 45744578.

14. Nagy, I., and Floyd, R.A. (1984): Hydroxyl free radical reactions with amino acids and proteins studied by electron spin resonance spectroscopy and spin-trapping. Biochim. Biophys. Acta, 790, 238-250. 